\title{
Relasi Ekuivalen Serupa Semu pada Ring Reguler Stable Diperumum
}

\author{
Evi Yuliza \\ Jurusan Matematika Fakultas MIPA Universitas Sriwijaya \\ Jalan Raya Palembang-Prabumulih KM.32, Indralaya 30662 \\ Email: evibc3@yahoo.com
}

\begin{abstract}
ABSTRAK
Dalam tulisan ini akan diselidiki relasi ekuivalen serupa semu pada ring reguler stable diperumum. Selanjutnya, akan diselidiki pula relasi ekuivalen serupa semu pada ring reguler stable diperumum dari himpunan bilangan bulat $n$. Awalnya, akan diselidiki relasi serupa semu bersifat refleksif pada ring reguler stable diperumum, relasi serupa semu bersifat simetris pada ring reguler stable diperumum dan relasi serupa semu bersifat transitif pada ring reguler stable diperumum. Kemudian, diselidiki relasi serupa semu bersifat refleksif pada ring reguler stable diperumum dari himpunan bilangan bulat $n$, relasi serupa semu bersifat simetris pada ring reguler stable diperumum dari himpunan bilangan bulat $n$ dan relasi serupa semu bersifat transitif pada ring reguler stable diperumum dari himpunan bilangan bulat $n$.
\end{abstract}

Kata kunci: similar semu, ring reguler, ring stable diperumum.

\begin{abstract}
In this paper will investigate the pseudo similar equivalence relation to the generalized stable regular ring. Furthermore, the relation will be investigated also pseudo similar equivalent to the regular ring of stable generalized set of integers $n$. Initially, it will be investigated the pseudo similar relationship is reflexive on generalized stable regular ring, the pseudo similar relationship is symmetrical on generalized stable regular ring and the pseudo similar relationship is transitive on stable generalized regular ring. Then, investigated the relation of similar apparent is reflexive in the generalized stable ring regular from the set of integers $n$, a relation the pseudo similar relationship is symmetrical to the generalized stable regular ring from the set of integers $n$ and the pseudo similar relationship is transitive on the generalized stable regular ring from the set of integers $n$.
\end{abstract}

Keywords: pseudo similarity, ring reguler, generalized stable ring.

\section{Pendahuluan}

Suatu ring $R$ dikatakan memenuhi sifat stable range one apabila memenuhi kondisi berikut: jika $a R+b R=R$ maka terdapat suatu $y \in R$ sehingga $a+b y \in U(R)$ (Chen, [1]) dengan $U(R)$ himpunan semua unit dalam $R$ (Goodearl, [6]). Dalam hal ini, ring $R$ yang dimaksud adalah ring komutatif dengan elemen satuan. Suatu ring $R$ dikatakan ring reguler apabila untuk setiap $x$ di $R$ ada $y$ di $R$ sehingga $x=$ $x y x$. (Goodearl et al, [5]) Goodearl [6] menyelidiki keterkaitan antara ring reguler yang memenuhi sifat stable range one dengan unit reguler, yaitu: Jika $R$ ring reguler memenuhi sifat stable range one maka $R$ merupakan unit ring reguler. Dalam penelitian Yuliza [7] memberikan bukti yang lebih sederhana dan mendasar mengenai keterkaitan antara ring reguler dengan stable range one. Beberapa peneliti telah menyelidiki ring yang memenuhi stable range one. Di antaranya adalah Chen [2], Goodearl et al [5]. Keterkaitan antara ring reguler $R$ dengan sifat ring stable range one dikemukakan oleh Chen [3]. Chen et al [4] memberikan bukti yang lain yang menunjukkan keterkaitan antara ring reguler yang memenuhi sifat stable range one dengan unit reguler.

Melalui $U(R)$ dapat dibentuk himpunan yang lebih umum yaitu $K(R)$ dengan definisi sebagai berikut, $K(R)=\{x \in R \mid(\exists s, t \in R)$ sxt $=1\} . K(R)$ adalah generalisasi dari $U(R)$, sebab: jika $x \in U(R)$ maka terdapat $u \in R$ sehingga $u x=1$ yang berarti $u x .1=1$. Dari ring $R$ yang memenuhi sifat stable range one dapat didefinisikan ring stable diperumum sebagai berikut: Suatu ring $R$ merupakan ring stable diperumum jika $a R+b R=R$ maka $a+b y \in K(R)$ untuk suatu $y \in R$. Yuliza [7] telah menyelidiki keterkaitan antara ring reguler dengan ring stable diperumum. Keterkaitan antara ring reguler $R$ dengan sifat ring stable diperumum dikemukakan oleh Chen [3] berikut ini: Ring reguler stable diperumum jika dan hanya jika untuk setiap $x \in R$ terdapat suatu $w \in K(R)$ dan suatu grup $G$ di $R$ sehingga $w x \in G$. Yuliza [7] menyelidiki bahwa: Ring reguler stable diperumum jika dan hanya jika untuk setiap $x \in R$ terdapat suatu $w \in K(R)$ dan suatu grup $G$ di $R$ sehingga $w x \in G$. 
Penelitian ini merupakan lanjutan penelitian sebelumnya yang telah dilakukan oleh Yuliza [8] yang berkaitan dengan sifat-sifat serupa semu pada ring reguler stable diperumum. Yuliza [9] telah menyelidiki ring reguler stable diperumum pada himpunan bilangan bulat modulo $n$. Pengembangan berikutnya yang akan dilakukan dalam penelitian ini adalah menyelidiki relasi ekuivalen serupa semu pada ring reguler stable diperumum. Kemudian pada penelitian ini akan diselidiki relasi serupa semu bersifat refleksif pada ring reguler stable diperumum dari himpunan bilangan bulat $n$, relasi serupa semu bersifat simetris pada ring reguler stable diperumum dari himpunan bilangan bulat $n$ dan relasi serupa semu bersifat transitif pada ring reguler stable diperumum dari himpunan bilangan bulat $n$.

\section{Metode Penelitian}

Langkah-langkah yang akan dilakukan dalam penelitian ini adalah:

1. Mendefinisikan similar semu atas ring reguler stable diperumum.

2. Menyelidiki relasi simetris pada similar semu atas ring reguler stable diperumum.

3. Menyelidiki relasi refleksif pada similar semu atas ring reguler stable diperumum.

4. Menyelidiki relasi transitif pada similar semu atas ring reguler stable diperumum.

5. Menyelidiki relasi simetris pada similar semu atas ring reguler stable diperumum dari himpunan bilangan bulat modulo $n$.

6. Menyelidiki relasi refleksif pada similar semu atas ring reguler stable diperumum dari himpunan bilangan bulat modulo $n$.

7. Menyelidiki relasi transitif pada similar semu atas ring reguler stable diperumum dari himpunan bilangan bulat modulo $n$.

8. Interpreasi hasil.

\section{Hasil dan Pembahasan}

Didefinisikan ring $R$ adalah ring komutatif dengan elemen satuan. Misalkan $a, b \in R$. Elemen $a$ dikatakan berelasi serupa semu terhadap $b$ di $R$ apabila terdapat $x, y, z \in R$ sehingga $x a y=b, \quad z b x=a, x y x=x z x=x$ dan dinotasikan dengan $a \approx b$.(Chen, [3])

Lemma 1. Jika $R$ ring dan $a, b \in R$ maka pernyataan berikut ekuivalen:

(1) $a \approx b$.

(2) Terdapat $x, y \in R$ sehingga $a=x b y, b=y a x, x=x y x$ dan $y=y x y$.

(Chen, [3])

\section{Bukti:}

(1) $\Rightarrow$ (2)

Diketahui $a \approx b$ yang berarti $a$ merupakan serupa semu terhadap $b$ di $R$ maka terdapat $x, y, z \in R$ sehingga yax $=b, x b z=a, x y x=x z x=x$. Ambil $y=z$. Dari sini diperoleh, $y(x y x) y=y(x z x) y=y x y=y$. Jadi, jika $a \approx b$ terdapat $x, y \in R$ sehingga $a=x b y, b=y a x, x=x y x$ dan $y=y x y$.

$(2) \Rightarrow(1)$

Misalkan terdapat $x, y, z \in R$ sehingga $b=x a y, a=z b x$ dan $x=x y x=x z x$. Selanjutnya,

$x a(y x y)=x a y=b,(z x z) b x=z b x=a$,

Menurut yang diketahui, diperoleh

$x(y x y) x=x y x=x=x z x=x(z x z) x,(y x y) x(y x y)=y x y$ dan $(z x z) x(z x z)=z x z$.

Dengan kata lain, $y=y x y$ dan $z=z x z$. Oleh karena itu, $x a(z x y)=x a y=b,(z x y) b x=z b x=a, z x y=(z x y) x(z x y)$ dan

$x=x(z x y) x$. Jadi, $a \approx b$.

Misalkan $R$ ring reguler stable diperumum dan $a, b \in R$. Elemen $a$ dikatakan berelasi serupa semu terhadap $b$ di $R$ apabila terdapat $x, y, z \in R$ sehingga $x a y=b, z b x=a, \quad x y x=x z x=x$ dan dinotasikan dengan $a \approx b$.

Akan ditunjukkan relasi serupa semu bersifat simetris pada ring reguler stable diperumum yakni, $a \approx b$ maka $b \bar{\sim}$. Diketahui $a \bar{\approx} b$ yang berarti $a$ merupakan serupa semu terhadap $b$ di $R$ maka terdapat $x, y, z \in R$ sehingga $y a x=b, x b z=a, x y x=x z x=x$. Ambil $y=z$. Menurut Lemma 1, jika $a \approx b$ terdapat $x, y \in R$ sehingga $a=$ $x b y, b=y a x, x=x y x$ dan $y=y x y$. 


$$
\begin{gathered}
a=x b y \\
(y x) a(y x)=(y x) x b y(y x) \\
y(x a y) x=(x y) x b y(x y) \\
y b x=(x y) b(y x y) \\
y b x=x b y=a . \\
\text { diperoleh } y b x=a . \quad y a x \\
b=y(x y)=(x y) y a x(x y) \\
(x y) b b x) y=(y x) y a x(y x) \\
x a y=(y x y) a(x y x) \\
x a y=y a x=b
\end{gathered}
$$

diperoleh $x a y=b$.

Jadi, relasi serupa semu bersifat simetris dipenuhi yang berarti $b$ merupakan serupa semu terhadap $a$, yakni $b \approx$ $a$ terdapat $x, y \in R$ sehingga $a=y b x, b=x a y, x=x y x$ dan $y=y x y$.

Akan ditunjukkan relasi serupa semu bersifat refleksif pada ring reguler stable diperumum yakni, $a \approx a$. Menurut Lemma 1, ambil $a=b$ sehingga $a=x a y$.

$$
\begin{gathered}
(y x) a(y x)=(y x) x a y(y x) \\
y(x a y) x=(x y) x a y(x y) \\
y a x=(x y x) a(y x y) \\
y a x=x a y=a
\end{gathered}
$$

Jadi, $a \approx a$ yang berarti relasi serupa semu bersifat refleksif dipenuhi yakni $a$ serupa semu terhadap $a$ terdapat $x, y \in R$ sehingga $a=x a y, a=y a x, x=x y x$ dan $y=y x y$.

Akan ditunjukkan relasi serupa semu bersifat transitif pada ring reguler stable diperumum yakni, $a \approx b$ dan $b \approx$ $c$ maka $a \approx c$. Menurut Lemma 1, jika $a \approx b$ terdapat $x, y \in R$ sehingga $a=x b y, b=y a x, x=x y x$ dan $y=y x y$. Selain itu, menurut Lemma 1 jika $b \approx c$ terdapat $x, y \in R$ sehingga $b=x c y, c=y b x, x=x y x$ dan $y=y x y$.

$$
\begin{aligned}
a & =x b y \\
& =x(x c y) y=x^{\prime} c y^{\prime} \text { dengan } x^{\prime}=x x, y^{\prime}=y y \text { dan } x^{\prime}, y^{\prime} \in R \\
c & =y b x \\
& =y(y a x) x=y^{\prime} a x^{\prime} \text { dengan } y^{\prime}=y y, x^{\prime}=x x \text { dan } x^{\prime}, y^{\prime} \in R
\end{aligned}
$$

diperoleh $a \approx c$ terdapat $x, y \in R$ sehingga $a=x c y, c=y a x, x=x y x$ dan $y=y x y$. Jadi, relasi serupa semu bersifat transitif pada ring reguler stable diperumum dipenuhi. Dengan demikian, relasi serupa semu bersifat refleksif pada ring reguler stable diperumum, relasi serupa semu bersifat simetris pada ring reguler stable diperumum dan relasi serupa semu bersifat transitif pada ring reguler stable diperumum dipenuhi .

Selanjutnya, relasi serupa semu bersifat refleksif pada ring reguler stable diperumum dari himpunan bilangan bulat $n$, relasi serupa semu bersifat simetris pada ring reguler stable diperumum dari himpunan bilangan bulat $n$ dan relasi serupa semu bersifat transitif pada ring reguler stable diperumum dari himpunan bilangan bulat $n$. Dalam hal ini, himpunan bilangan bulat modulo $n$ yang dimaksud adalah himpunan bilangan bulat modulo $n$ bukan nol.

Ring reguler yang memenuhi stable diperumum pada himpunan bilangan bulat modulo $n$ (Yuliza, [9]) apabila $n$ prima. Untuk ring reguler stable diperumum $\mathbb{Z}_{2}$ dan $\mathbb{Z}_{3}$, jelas memenuhi relasi refleksif dan relasi simetris pada similar semu atas ring reguler stable diperumum. Di definisikan $a$ merupakan similar semu terhadap $b$ di $\mathbb{Z}_{5}$, jika $a \approx b$ terdapat $x, y \in \mathbb{Z}_{5}$ sehingga $a=x b y, b=y a x, x=x y x$ dan $y=y x y$.

Ambil $\overline{1}, \overline{1} \in \mathbb{Z}_{5}$, jelas bahwa $\overline{1} \approx \overline{1}$.

Ambil $\overline{1}, \overline{2} \in \mathbb{Z}_{5}, \overline{1} \neq \overline{2}$, sebab tidak terdapat $x, \mathrm{y} \in \mathbb{Z}_{5}$ sehingga $\overline{1}=x \overline{2} y, \overline{2}=y \overline{1} x, \overline{1}=\overline{1} \cdot \overline{1} \cdot \overline{1}$ dan $\overline{2}=\overline{2} \cdot \overline{3} . \overline{2}$. Ambil $\overline{1}, \overline{3} \in \mathbb{Z}_{5}, \overline{1} \nsucc \overline{3}$, sebab tidak terdapat $x, y \in \mathbb{Z}_{5}$ sehingga $\overline{1}=x \overline{3} y, \overline{3}=y \overline{1} x, \overline{1}=\overline{1} . \overline{1} \cdot \overline{1}$ dan $\overline{3}=\overline{3} 2 \overline{3}$. Ambil $\overline{1}, \overline{4} \in \mathbb{Z}_{5}, \overline{1} \approx \overline{4}$, sebab terdapat $\overline{1}, \overline{4} \in \mathbb{Z}_{5}$ sehingga $\overline{1}=\overline{4} \cdot \overline{4} \cdot \overline{1}, \overline{4}=\overline{1} \cdot \overline{1} \cdot \overline{4}, \overline{1}=\overline{1} \cdot \overline{1} \cdot \overline{1}$ dan $\overline{4}=\overline{4} \cdot \overline{4} \cdot \overline{4}$ Ambil $\overline{2}, \overline{2} \in \mathbb{Z}_{5}, \overline{2} \approx \overline{2}$, sebab terdapat $\overline{2}, \overline{3} \in \mathbb{Z}_{5}$ sehingga $\overline{2}=\overline{2} \cdot \overline{2} \cdot \overline{3}, \overline{3}=\overline{3} \cdot \overline{2} \cdot \overline{2}, \overline{2}=\overline{2} \cdot \overline{3} \cdot \overline{2}$ dan $\overline{3}=\overline{3} \cdot \overline{2} \cdot \overline{3}$. Ambil $\overline{2}, \overline{3} \in \mathbb{Z}_{5}, \overline{2} \approx \overline{3}$, sebab terdapat $\overline{1}, \overline{4} \in \mathbb{Z}_{5}$ sehingga $\overline{2}=\overline{1} \cdot \overline{3} \cdot \overline{4}, \overline{3}=\overline{4} \cdot \overline{2} \cdot \overline{1}, \overline{2}=\overline{2} \cdot \overline{3} \cdot \overline{2}$ dan $\overline{3}=\overline{3} \cdot \overline{2} \cdot \overline{3}$. Ambil $\overline{2}, \overline{4} \in \mathbb{Z}_{5}, \overline{2} \neq \overline{4}$, sebab tidak terdapat $x, y \in \mathbb{Z}_{5}$ sehingga $\overline{2}=x \overline{4} y, \overline{4}=y \overline{2} x, \overline{2}=\overline{2} \cdot \overline{3} \cdot \overline{2}$ dan $\overline{4}=\overline{4} \cdot \overline{4} \cdot \overline{4}$. Ambil $\overline{3}, \overline{1} \in \mathbb{Z}_{5}, \overline{3} \neq \overline{1}$, sebab tidak terdapat $x, y \in \mathbb{Z}_{5}$ sehingga $3=x \overline{1} y, \overline{1}=y \overline{3} x, \overline{3}=\overline{3} \cdot \overline{2} \cdot \overline{3}$ dan $\overline{1}=\overline{1} \cdot \overline{1} \cdot \overline{1}$. Ambil $\overline{3}, \overline{2} \in \mathbb{Z}_{5}, \overline{3} \approx \overline{2}$, sebab terdapat $\overline{1}, \overline{4} \in \mathbb{Z}_{5}$ sehingga $\overline{3}=\overline{1} \cdot \overline{2} \cdot \overline{4}, \overline{2}=\overline{4} \cdot \overline{3} \cdot \overline{1}, \overline{3}=\overline{3} \cdot \overline{2} \cdot \overline{3}$ dan $\overline{2}=\overline{2} \cdot \overline{3} \cdot \overline{2}$. 
Ambil $\overline{3}, \overline{3} \in \mathbb{Z}_{5}, \overline{3} \approx \overline{3}$, sebab terdapat $\overline{2}, \overline{3} \in \mathbb{Z}_{5}$ sehingga $\overline{3}=\overline{2} \cdot \overline{3} \cdot \overline{3}, \overline{3}=\overline{3} \cdot \overline{3} \cdot \overline{2}, \overline{2}=\overline{2} \cdot \overline{3} \cdot \overline{2}$ dan $\overline{3}=\overline{3} \cdot \overline{2} \cdot \overline{3}$. Ambil $\overline{3}, \overline{4}, \in \mathbb{Z}_{5}, \overline{3} \nsucc \overline{4}$, , sebab tidak terdapat $x, y \in \mathbb{Z}_{5}$ sehingga $\overline{3}=x \overline{4} y, \overline{4}=y \overline{3} x, \overline{3}=\overline{3} \cdot \overline{2} \cdot \overline{3}$ dan $\overline{4}=\overline{4} \cdot \overline{4} . \overline{4}$. Ambil $\overline{4}, \overline{1} \in \mathbb{Z}_{5}, \overline{4} \approx \overline{1}$, sebab terdapat $\overline{1}, \overline{4} \in \mathbb{Z}_{5}$ sehingga $\overline{4}=\overline{4} \cdot \overline{1} \cdot \overline{1}, \overline{1}=\overline{1} \cdot \overline{4} \cdot \overline{4}, \overline{1}=\overline{1} \cdot \overline{1} \cdot \overline{1}$ dan $\overline{4}=\overline{4} \cdot \overline{4} \cdot \overline{4}$. Ambil $\overline{4}, \overline{2} \in \mathbb{Z}_{5}, \overline{4} \nsucc \overline{2}$, sebab tidak terdapat $x, y \in \mathbb{Z}_{5}$ sehingga $\overline{4}=x \overline{2} y, \overline{2}=y \overline{4} x, \overline{4}=\overline{4} . \overline{4} . \overline{4}$ dan $\overline{2}=\overline{2} . \overline{3} . \overline{2}$. Ambil $\overline{4}, \overline{3} \in \mathbb{Z}_{5}, \overline{4} \neq \overline{3}$, sebab tidak terdapat $x, y \in \mathbb{Z}_{5}$ sehingga $\overline{4}=y \overline{3} x, \overline{3}=x \overline{4} y, \overline{3}=\overline{3} \cdot \overline{2} \cdot \overline{3}$ dan $\overline{4}=\overline{4} . \overline{4} . \overline{4}$. Ambil $\overline{4}, \overline{4} \in \mathbb{Z}_{5}, \overline{4} \approx \overline{4}$, sebab terdapat $\overline{2}, \overline{3} \in \mathbb{Z}_{5}$ sehingga $\overline{4}=\overline{2} \cdot 4 \cdot \overline{3}, \overline{4}=\overline{3} . \overline{4} . \overline{2}, \overline{4}=\overline{4} . \overline{4} . \overline{4}$ dan $\overline{4}=\overline{4} . \overline{4} . \overline{4}$.

Akan ditunjukkan relasi serupa semu bersifat refleksif pada ring reguler stable diperumum $\mathbb{Z}_{5}$. Di definisikan relasi serupa semu bersifat refleksif pada ring reguler stable diperumum $\mathbb{Z}_{5}$, yakni, $a \approx a$ untuk setiap $a \in \mathbb{Z}_{5}$. Jelas bahwa $\overline{1} \approx \overline{1}$.

$\overline{2} \approx \overline{2}$, sebab terdapat $\overline{2}, \overline{3} \in \mathbb{Z}_{5}$ sehingga $\overline{2}=\overline{2} \cdot \overline{2} \cdot \overline{3}, \overline{3}=\overline{3} \cdot \overline{2} \cdot \overline{2}, \overline{2}=\overline{2} \cdot 3 \cdot \overline{2}$ dan $\overline{3}=\overline{3} \cdot \overline{2} \cdot \overline{3}$.

$\overline{3} \approx \overline{3}$, sebab terdapat $\overline{2}, \overline{3} \in \mathbb{Z}_{5}$ sehingga $\overline{3}=\overline{2} \overline{3} \cdot \overline{3}, \overline{3}=\overline{3} \cdot \overline{3} \cdot \overline{2}, \overline{2}=\overline{2} \cdot \overline{3} \cdot \overline{2}$ dan $\overline{3}=\overline{3} \cdot \overline{2} \cdot \overline{3}$.

$\overline{4} \approx \overline{4}$, sebab terdapat $\overline{2}, \overline{3} \in \mathbb{Z}_{5}$ sehingga $\overline{4}=\overline{2} \cdot \overline{4} \cdot \overline{3}, \overline{4}=\overline{3} \cdot \overline{4} \cdot \overline{2}, \overline{4}=\overline{4} \cdot \overline{4} . \overline{4}$ dan $\overline{4}=\overline{4} . \overline{4} . \overline{4}$

Jadi, relasi serupa semu bersifat refleksif pada ring reguler stable diperumum $\mathbb{Z}_{5}$ dipenuhi.

Akan ditunjukkan relasi serupa semu bersifat simetris pada ring reguler stable diperumum $\mathbb{Z}_{5}$. Di definisikan relasi similar semu bersifat simetris pada ring reguler ring reguler stable diperumum $\mathbb{Z}_{5}$, yakni, $a \approx b$ maka $b \bar{\approx}$ $a$. Jika $a \approx b$ terdapat $x, y \in R$ sehingga $a=x b y, b=y a x, x=x y x$ dan $y=y x y$. Di peroleh, $b \approx a$ terdapat $x, y \in$ $\mathbb{Z}_{5}$ sehingga $a=y b x, b=x a y, x=x y x$ dan $y=y x y$.

Jika $\overline{1} \approx \overline{4}$ terdapat $\overline{1}, \overline{4} \in \mathbb{Z}_{5}$ sehingga $\overline{1}=\overline{4} \cdot \overline{4} \cdot \overline{1}, \overline{4}=\overline{1} \cdot \overline{1} \cdot \overline{4}, \overline{1}=\overline{1} \cdot \overline{1} \cdot \overline{1}$ dan $\overline{4}=\overline{4} \cdot \overline{4} \cdot \overline{4}$, maka $\overline{4} \approx \overline{1}$ terdapat $\overline{1}, \overline{4} \in \mathbb{Z}_{5}$ sehingga $\overline{4}=\overline{4} \cdot \overline{1} \cdot \overline{1}, \overline{1}=\overline{1} \cdot \overline{4} \cdot \overline{4}, \overline{1}=\overline{1} \cdot \overline{1} \cdot \overline{1}$ dan $\overline{4}=\overline{4} \cdot \overline{4} \cdot \overline{4}$. Jika $\overline{2} \approx \overline{3}$ terdapat $\overline{1}, \overline{4} \in \mathbb{Z}_{5}$ sehingga $\overline{2}=\overline{1} \cdot 3 \overline{4}, \overline{3}=\overline{4} \cdot \overline{2} \cdot \overline{1}, \overline{2}=\overline{2} \cdot 3 \cdot \overline{2}$ dan $\overline{3}=\overline{3} \cdot \overline{2} \cdot \overline{3}$ maka $\overline{3} \approx \overline{2}$ terdapat $\overline{1}, \overline{4} \in \mathbb{Z}_{5}$ sehingga $\overline{3}=\overline{1} \cdot \overline{2} \cdot \overline{4}, \overline{2}=$ $\overline{4} . \overline{3} \cdot \overline{1}, \overline{3}=\overline{3} \cdot \overline{2} \cdot \overline{3}$ dan $\overline{2}=\overline{2} \cdot \overline{3} \cdot \overline{2}$.

Akan ditunjukkan ring serupa semu bersifat transitif pada ring reguler stable diperumum $\mathbb{Z}_{5}$. Jika $\overline{1} \approx \overline{4}$ dan $\overline{4}$ $\bar{\sim}$ maka akan ditunjukkan $\overline{1} \approx \overline{1}$.

$\overline{1} \approx \overline{4}$ berarti terdapat $1, \overline{4} \in \mathbb{Z}_{5}$ sehingga $\overline{1}=\overline{4} \cdot \overline{4} \cdot \overline{1}, \overline{4}=\overline{1} \cdot \overline{1} \cdot \overline{4}, \overline{1}=\overline{1} \cdot \overline{1} \cdot \overline{1}$ dan $\overline{4}=\overline{4} \cdot \overline{4} \cdot \overline{4}$.

$\overline{4}=\overline{1}$ berarti terdapat $\overline{1}, \overline{4} \in \mathbb{Z}_{5}$ sehingga $\overline{4}=\overline{4} \cdot \overline{1} \overline{1}, \overline{1}=\overline{1} \cdot \overline{4} \cdot \overline{4}, \overline{1}=\overline{1} \cdot \overline{1} \cdot \overline{1}$ dan $\overline{4}=\overline{4} \cdot \overline{4} \cdot \overline{4}$.

Diperoleh $\overline{1}=\overline{4} \cdot(\overline{4} \cdot \overline{1} \cdot \overline{1}) \cdot \overline{1}=\overline{1} \cdot \overline{1} \cdot \overline{1}, \overline{1}=\overline{1} \cdot(\overline{1} \cdot \overline{1} \cdot \overline{4}) \cdot \overline{4}=\overline{1} \cdot \overline{1} \cdot \overline{1}$ yang berarti $\overline{1} \approx \overline{1}$.

Jadi, relasi serupa semu bersifat transtifs pada ring reguler stable diperumum $\mathbb{Z}_{5}$ dipenuhi.

Didefinisikan $a$ merupakan similar semu terhadap $b$ di $\mathbb{Z}_{7}$, jika $a \approx b$ terdapat $x, y \in \mathbb{Z}_{7}$ sehingga $a=x b y$, $b=y a x, x=x y x$ dan $y=y x y$.

Ambil $\overline{1}, \overline{1} \in \mathbb{Z}_{7}$, jelas bahwa $\overline{1} \approx \overline{1}$.

Ambil $\overline{1}, \overline{2} \in \mathbb{Z}_{7}, \overline{1} \nsucc \overline{2}$, sebab tidak terdapat $x, y \in \mathbb{Z}_{7}$ sehingga $\overline{1}=x \overline{2} y, 2=y \overline{1} x, \overline{1}=\overline{1} \cdot \overline{1} \cdot \overline{1}$ dan $\overline{2}=\overline{2} . \overline{4} \cdot \overline{2}$.

Ambil $\overline{1}, \overline{3} \in \mathbb{Z}_{7}, \overline{1} \neq \overline{3}$, sebab tidak terdapat $x, y \in \mathbb{Z}_{7}$ sehingga $\overline{1}=x \overline{3} y, \overline{3}=y \overline{1} x, \overline{1}=\overline{1} \cdot \overline{1} \cdot \overline{1}$ dan $\overline{3}=\overline{3} . \overline{5} . \overline{3}$.

Ambil $\overline{1}, \overline{4} \in \mathbb{Z}_{7}, \overline{1} \neq \overline{4}$, sebab tidak terdapat $x, y \in \mathbb{Z}_{7}$ sehingga $\overline{1}=x \overline{4} y, \overline{4}=y \overline{1} x, \overline{1}=\overline{1} \cdot \overline{1} \cdot \overline{1}$ dan $\overline{4}=\overline{4} . \overline{2} . \overline{4}$.

Ambil $\overline{1}, 5 \in \mathbb{Z}_{7}, \overline{1} \neq \overline{5}$, sebab tidak terdapat $x, y \in \mathbb{Z}_{7}$ sehingga $\overline{1}=x \overline{5} y, \overline{5}=y \overline{1} x, \overline{1}=\overline{1} . \overline{1} \cdot \overline{1}$ dan $\overline{5}=\overline{5} . \overline{3} . \overline{5}$.

Ambil $\overline{1}, 6 \in \mathbb{Z}_{7}, \overline{1} \approx \overline{6}$, sebab terdapat $\overline{2}, \overline{3} \in \mathbb{Z}_{7}$ sehingga $\overline{1}=\overline{2} \cdot 6 \cdot \overline{3}, \overline{6}=\overline{3} \cdot \overline{1} \cdot \overline{2}, \overline{1}=\overline{1} \cdot \overline{1} \cdot \overline{1}$ dan $\overline{6}=\overline{6} \cdot \overline{6} \cdot \overline{6}$.

Ambil $\overline{2}, \overline{2} \in \mathbb{Z}_{7}, \overline{2} \approx \overline{2}$, sebab terdapat $\overline{3}, \overline{5} \in \mathbb{Z}_{7}$ sehingga $\overline{2}=\overline{3} \cdot \overline{2} \cdot \overline{5}, \overline{2}=\overline{5} \cdot \overline{2} \cdot \overline{3}$ dan $\overline{2}=\overline{2} \cdot \overline{4} \cdot \overline{2}$.

Ambil $\overline{2}, \overline{3} \in \mathbb{Z}_{7}, \overline{2} \nsucc \overline{3}$, sebab tidak terdapat $x, y \in \mathbb{Z}_{7}$ sehingga $\overline{2}=x \overline{3} y, \overline{3}=y \overline{2} x, \overline{2}=\overline{2} . \overline{4} . \overline{2}$ dan $\overline{3}=\overline{3} . \overline{5} . \overline{3}$.

Ambil $\overline{2}, \overline{4} \in \mathbb{Z}_{7}, \overline{2} \nsucc \overline{4}$, sebab tidak terdapat $x, y \in \mathbb{Z}_{7}$ sehingga $\overline{2}=x \overline{4} y, \overline{4}=y \overline{2} x, \overline{2}=\overline{2} . \overline{4} . \overline{2}$ dan $\overline{4}=\overline{4} .2 . \overline{4}$.

Ambil $\overline{2}, \overline{5} \in \mathbb{Z}_{7}, \overline{2} \neq \overline{5}$, sebab tidak terdapat $x, y \in \mathbb{Z}_{7}$ sehingga $\overline{2}=x \overline{5} y, \overline{5}=y \overline{2} x, \overline{2}=\overline{2} \cdot \overline{4} \cdot \overline{2}$ dan $\overline{5}=\overline{5} . \overline{3} . \overline{5}$.

Ambil $\overline{2}, 6 \in \mathbb{Z}_{7}, \overline{2} \nsucc \overline{6}$, sebab tidak terdapat $x, y \in \mathbb{Z}_{7}$ sehingga $\overline{2}=x \overline{6} y, \overline{6}=y \overline{2} x, \overline{2}=\overline{2} \cdot \overline{4} \cdot \overline{2}$ dan $\overline{6}=\overline{6} \cdot \overline{6} \cdot \overline{6}$.

Ambil $\overline{3}, \overline{3} \in \mathbb{Z}_{7}, \overline{3}=\overline{3}$, sebab terdapat $\overline{2}, \overline{4} \in \mathbb{Z}_{7}$ sehingga $\overline{3}=\overline{2} \cdot \overline{3} \cdot \overline{4}, \overline{3}=4 . \overline{3} \cdot \overline{2}, \overline{2}=\overline{2} \cdot \overline{4} \cdot \overline{2}$ dan $\overline{4}=\overline{4} \cdot \overline{2} \cdot \overline{4}$.

Ambil $\overline{3}, \overline{4} \in \mathbb{Z}_{7}, \overline{3} \approx \overline{4}$, sebab terdapat $\overline{2}, \overline{3} \in \mathbb{Z}_{7}$ sehingga $\overline{3}=\overline{2} \cdot 4 \cdot \overline{3}, \overline{4}=\overline{3} \cdot \overline{3} \cdot \overline{2}, \overline{3}=\overline{3} \cdot \overline{5} \cdot \overline{3}$ dan $\overline{4}=\overline{4} . \overline{2} . \overline{4}$.

Ambil $\overline{3}, 5 \in \mathbb{Z}_{7}, \overline{3} \nsucc \overline{5}$, sebab tidak terdapat $x, y \in \mathbb{Z}_{7}$ sehingga $\overline{3}=x \overline{5} y, \overline{5}=y \overline{3} x, \overline{3}=\overline{3} \cdot \bar{s} . \overline{3}$ dan $\overline{5}=\overline{5} . \overline{3} . \overline{5}$

Ambil $\overline{3}, \overline{6} \in \mathbb{Z}_{7}, \overline{3} \neq \overline{6}$, sebab tidak terdapat $x, y \in \mathbb{Z}_{7}$ sehingga $\overline{3}=x \overline{6} y, \overline{6}=y \overline{3} x, \overline{3}=\overline{3} . \overline{5} . \overline{3}$ dan $\overline{6}=\overline{6} . \overline{6} \cdot \overline{6}$

Ambil $\overline{4}, \overline{1} \in \mathbb{Z}_{7}, \overline{4} \neq \overline{1}$, sebab tidak terdapat $x, y \in \mathbb{Z}_{7}$ sehingga $\overline{4}=x \overline{1} y, \overline{1}=y \overline{4} x, \overline{4}=\overline{4} \cdot \overline{2} \cdot \overline{4}$ dan $\overline{1}=\overline{1} \cdot \overline{1} \cdot \overline{1}$.

Ambil $\overline{4}, \overline{2} \in \mathbb{Z}_{7}, \overline{4} \neq \overline{2}$, sebab tidak terdapat $x, y \in \mathbb{Z}_{7}$ sehingga $\overline{4}=x \overline{2} y, 2=y \overline{4} x, \overline{4}=\overline{4} \cdot \overline{2} . \overline{4}$ dan $\overline{2}=\overline{2} . \overline{4} . \overline{2}$.

Ambil $\overline{4}, \overline{3} \in \mathbb{Z}_{7}, \overline{4} \approx \overline{3}$ sebab terdapat $\overline{2}, \overline{3} \in \mathbb{Z}_{7}$ sehingga $\overline{4}=\overline{2} \cdot \overline{3} \cdot \overline{3}, \overline{3}=\overline{3} \cdot 4 \cdot \overline{3}, \overline{4}=\overline{4} . \overline{2} \overline{4}$ dan $\overline{3}=\overline{3} . \overline{5} . \overline{3}$.

Ambil $\overline{4}, \overline{4} \in \mathbb{Z}_{7}, \overline{4} \approx \overline{4}$ sebab terdapat $\overline{2}, \overline{4} \in \mathbb{Z}_{7}$ sehingga $\overline{4}=\overline{2} \cdot \overline{4} \cdot \overline{4}, \overline{4}=\overline{4} . \overline{4} . \overline{2}$ dan $\overline{4}=\overline{4} \cdot \overline{2} \overline{4}$.

Ambil $\overline{4}, \overline{5} \in \mathbb{Z}_{7}, \overline{4} \neq \overline{5}$, sebab tidak terdapat $x, y \in \mathbb{Z}_{7}$ sehingga $\overline{4}=x \overline{5} y, \overline{5}=y \overline{4} x, \overline{4}=\overline{4} . \overline{2} \overline{4}$ dan $\overline{5}=\overline{5} . \overline{3} . \overline{5}$

Ambil $\overline{4}, \overline{6} \in \mathbb{Z}_{7}, \overline{4} \neq \overline{6}$, sebab tidak terdapat $x, y \in \mathbb{Z}_{7}$ sehingga $\overline{4}=x \overline{6} y, \overline{6}=y 4 x, \overline{4}=\overline{4} \cdot \overline{2} \cdot \overline{4}$ dan $\overline{6}=\overline{6} \cdot \overline{6} \cdot \overline{6}$

Ambil $\overline{5}, \overline{1} \in \mathbb{Z}_{7}, \overline{5} \neq \overline{1}$, sebab tidak terdapat $x, y \in \mathbb{Z}_{7}$ sehingga $\overline{5}=x 1 y, \overline{1}=y \overline{5} x, \overline{5}=\overline{3} \cdot \overline{5} \cdot \overline{3}$ dan $\overline{1}=\overline{1} \cdot \overline{1} \cdot \overline{1}$. Ambil $\overline{5}, \overline{2} \in \mathbb{Z}_{7}, \overline{5} \nsucc \overline{2}$, sebab tidak terdapat $x, y \in \mathbb{Z}_{7}$ sehingga $\overline{5}=x \overline{2} y, \overline{2}=y \overline{5} x, \overline{5}=\overline{5} \cdot \overline{3} \cdot \overline{5}$ dan $\overline{2}=\overline{2} \cdot \overline{4} \cdot \overline{2}$. 
Ambil $\overline{5}, \overline{3} \in \mathbb{Z}_{7}, \overline{5} \nsim \overline{3}$, sebab tidak terdapat $x, y \in \mathbb{Z}_{7}$ sehingga $\overline{5}=x \overline{3} y, \overline{3}=y \overline{5} x, \overline{5}=\overline{5} \cdot \overline{3} \cdot \overline{5}$ dan $\overline{3}=\overline{3} \cdot \overline{5} \cdot \overline{3}$. Ambil $\overline{5}, \overline{4} \in \mathbb{Z}_{7}, \overline{5} \neq \overline{4}$, sebab tidak terdapat $x, y \in \mathbb{Z}_{7}$ sehingga $\overline{5}=x \overline{4} y, \overline{4}=y \overline{5} x, \overline{5}=\overline{5} .4 . \overline{5}$ dan $\overline{4}=\overline{4} . \overline{2} \overline{4}$. Ambil $\overline{5}, \overline{5} \in \mathbb{Z}_{7}, \overline{5} \approx \overline{5}$ sebab terdapat $\overline{3}, \overline{5} \in \mathbb{Z}_{7}$ sehingga $\overline{5}=\overline{3} \cdot \overline{5} \cdot \overline{5}, \overline{5}=\overline{5} \cdot \overline{5} \cdot \overline{3}$ dan $\overline{5}=\overline{5} \cdot \overline{3} \cdot \overline{5}$.

Ambil $\overline{5}, \overline{6} \in \mathbb{Z}_{7}, \overline{5} \neq \overline{6}$, sebab tidak terdapat $x, y \in \mathbb{Z}_{7}$ sehingga $\overline{5}=x 6 y, \overline{6}=y \overline{5} x, \overline{5}=\overline{5} \cdot \overline{3} \cdot \overline{5}$ dan $\overline{6}=\overline{6} \cdot \overline{6} \cdot \overline{6}$. Ambil $\overline{6}, \overline{1} \in \mathbb{Z}_{7}, \overline{6} \approx \overline{1}$ sebab terdapat $\overline{3}, \overline{2} \in \mathbb{Z}_{7}$ sehingga $\overline{6}=3 . \overline{1} \cdot \overline{2}, \overline{1}=\overline{2} \cdot \overline{6} \cdot \overline{3}, \overline{6}=\overline{6} \cdot \overline{6} \cdot \overline{6}$ dan $\overline{1}=\overline{1} \cdot \overline{1} \cdot \overline{1}$.

Ambil $\overline{6}, \overline{2} \in \mathbb{Z}_{7}, \overline{6} \nsucc \overline{2}$, sebab tidak terdapat $x, y \in \mathbb{Z}_{7}$ sehingga $\overline{6}=x 2 y, \overline{2}=y \overline{6} x, \overline{6}=\overline{6} \cdot \overline{6} \cdot \overline{6}$ dan $\overline{2}=\overline{2} \cdot \overline{4} \cdot \overline{2}$. Ambil $\overline{6}, \overline{3} \in \mathbb{Z}_{7}, \overline{6} \neq \overline{3}$ sebab tidak terdapat $x, y \in \mathbb{Z}_{7}$ sehingga $\overline{6}=x \overline{3} y, \overline{3}=y \overline{6} x, \overline{6}=\overline{6} \cdot \overline{6} \cdot \overline{6}$ dan $\overline{3}=\overline{3} \cdot \overline{5} \cdot \overline{3}$. Ambil $\overline{6}, \overline{5} \in \mathbb{Z}_{7}, \overline{6} \neq \overline{5}$, sebab tidak terdapat $x, y \in \mathbb{Z}_{7}$ sehingga $\overline{6}=x 5 y, \overline{5}=y \overline{6} x, \overline{6}=\overline{6} \cdot \overline{6} \cdot \overline{6}$ dan $\overline{5}=\overline{5} \cdot \overline{3} \cdot \overline{5}$. Ambil $\overline{6}, \overline{6} \in \mathbb{Z}_{7}, \overline{6} \approx \overline{6}$ sebab terdapat $\overline{3}, \overline{5} \in \mathbb{Z}_{7}$ sehingga $\overline{6}=\overline{3} \cdot \overline{6} \cdot \overline{5}, \overline{6}=\overline{5} \cdot \overline{6} \cdot \overline{3}$ dan $\overline{6}=\overline{6} \cdot \overline{6} \cdot \overline{6}$.

Akan ditunjukkan relasi serupa semu pada ring reguler stable diperumum $\mathbb{Z}_{7}$. Didefinisikan relasi serupa semu pada ring reguler stable diperumum $\mathbb{Z}_{7}$, yakni, $a \approx a$ untuk setiap $a \in \mathbb{Z}_{7}$.

Jelas bahwa $\overline{1} \approx \overline{1}$.

$\overline{2} \approx \overline{2}$, sebab terdapat $\overline{3}, \overline{5} \in \mathbb{Z}_{7}$ sehingga $\overline{2}=\overline{3} \cdot \overline{2} \cdot \overline{5}, \overline{2}=\overline{5} \cdot \overline{2} \cdot \overline{3}, \overline{3}=\overline{3} \cdot \overline{5} \cdot \overline{3}$ dan $\overline{5}=\overline{5} \cdot \overline{3} \cdot \overline{5}$.

$\overline{3} \approx \overline{3}$, sebab terdapat $\overline{2}, \overline{4} \in \mathbb{Z}_{7}$ sehingga $\overline{3}=\overline{2} \cdot \overline{3} \cdot \overline{4}, \overline{3}=\overline{4} \cdot \overline{3} \cdot \overline{2}, \overline{2}=\overline{2} \cdot \overline{4} \cdot \overline{2}$ dan $\overline{4}=\overline{4} \cdot \overline{2} \cdot \overline{4}$.

$\overline{4} \approx \overline{4}$, sebab terdapat $\overline{2}, \overline{4} \in \mathbb{Z}_{7}$ sehingga $\overline{4}=\overline{2} \cdot \overline{4} \cdot \overline{4}, \overline{4}=\overline{4} \cdot \overline{4} \cdot \overline{2}, \overline{2}=\overline{2} \cdot \overline{4} \cdot \overline{2}$ dan $\overline{4}=\overline{4} \cdot \overline{2} \cdot \overline{4}$.

$\overline{5} \approx \overline{5}$, sebab terdapat $\overline{3}, \overline{5} \in \mathbb{Z}_{7}$ sehingga $\overline{5}=\overline{3} \cdot \overline{5} \cdot \overline{5}, \overline{5}=\overline{5} \cdot \overline{5} \cdot \overline{3}, \overline{3}=\overline{3} \cdot \overline{5} \cdot \overline{3}$ dan $\overline{5}=\overline{5} \cdot \overline{3} \cdot \overline{5}$.

$\overline{6} \approx \overline{6}$, sebab terdapat $\overline{3}, \overline{5} \in \mathbb{Z}_{7}$ sehingga $\overline{6}=\overline{3} \cdot \overline{6} \cdot \overline{5}, \overline{6}=\overline{5} \cdot \overline{6} \cdot \overline{3}, \overline{3}=\overline{3} \cdot \overline{5} \cdot \overline{3}$ dan $\overline{5}=\overline{5} \cdot \overline{3} \cdot \overline{5}$.

Jadi, relasi serupa semu bersifat refleksif pada ring reguler stable diperumum $\mathbb{Z}_{7}$ dipenuhi.

Akan ditunjukkan relasi serupa semu bersifat simetris pada ring reguler stable diperumum $\mathbb{Z}_{7}$. Didefinisikan relasi simetris atas ring reguler stable diperumum $\mathbb{Z}_{7}$, yakni, $a \approx b$ maka $b \approx a$. Jika $a \approx b$ terdapat $x, y \in \mathbb{Z}_{7}$ sehingga $a=x b y, b=y a x, x=x y x$ dan $y=y x y$. Di peroleh, $b \approx a$ terdapat $x, y \in \mathbb{Z}_{7}$ sehingga $a=y b x, b=$ $x a y, x=x y x$ dan $y=y x y$.

Jika $\overline{3} \approx \overline{4}$ terdapat $\overline{2}, \overline{3} \in \mathbb{Z}_{7}$ sehingga $\overline{3}=\overline{2} \cdot \overline{4} \cdot \overline{3}, \overline{4}=\overline{3} \cdot \overline{3} \cdot \overline{2}, \overline{3}=\overline{3} \cdot \overline{5} \cdot \overline{3}$ dan $\overline{4}=\overline{4} \cdot \overline{2} \cdot \overline{4}$ maka $\overline{4} \approx \overline{3}$ terdapat $\overline{2}, \overline{3} \in \mathbb{Z}_{7}$ sehingga $\overline{4}=\overline{2} \cdot \overline{3} \cdot \overline{3}, \overline{4}=\overline{3} \cdot \overline{4} \cdot \overline{3}, \overline{4}=\overline{4} \cdot \overline{2} \cdot \overline{4}$ dan $\overline{3}=\overline{3} \cdot \overline{5} \cdot \overline{3}$. Jika $\overline{1} \approx \overline{6}$, terdapat $\overline{2}, \overline{3} \in \mathbb{Z}_{7}$ sehingga $\overline{1}=\overline{2} \cdot \overline{6} \cdot \overline{3}, \overline{6}=\overline{3} \cdot \overline{1} \cdot \overline{2}, \overline{1}=\overline{1} \cdot \overline{1} \cdot \overline{1}$ dan $\overline{6}=\overline{6} \cdot \overline{6} \cdot \overline{6}$ maka $\overline{6}=\overline{1}$ terdapat $\overline{3}, \overline{2} \in \mathbb{Z}_{7}$ sehingga $\overline{6}=\overline{3} \cdot \overline{1} \cdot \overline{2}, \overline{1}=$ $\overline{2} \overline{6} \cdot \overline{3}, \overline{6}=\overline{6} \cdot \overline{6} \cdot \overline{6}$ dan $\overline{1}=\overline{1} \cdot \overline{1} \cdot \overline{1}$ Jadi, relasi serupa semu bersifat transitif pada reguler stable diperumum $\mathbb{Z}_{7}$ dipenuhi.

Akan ditunjukkan relasi serupa semu bersifat transitif pada ring reguler stable diperumum $\mathbb{Z}_{7}$. Jika $\overline{1} \approx \overline{4}$ dan $\overline{4} \approx \overline{1}$ maka akan ditunjukkan $\overline{1} \approx \overline{1}$.

$\overline{1} \approx \overline{4}$ berarti terdapat $\overline{1}, \overline{4} \in \mathbb{Z}_{5}$ sehingga $\overline{1}=\overline{4} \cdot \overline{4} \cdot \overline{1}, \overline{4}=\overline{1} \cdot \overline{1} \overline{4}, \overline{1}=\overline{1} \cdot \overline{1} \cdot \overline{1}$ dan $\overline{4}=\overline{4} \cdot \overline{4} \cdot \overline{4}$.

$\overline{4} \approx \overline{1}$ berarti terdapat $\overline{1}, \overline{4} \in \mathbb{Z}_{5}$ sehingga $\overline{4}=\overline{4} \cdot \overline{1} \cdot \overline{1}, \overline{1}=\overline{1} \cdot \overline{4} \cdot \overline{4}, \overline{1}=\overline{1} \cdot \overline{1} \cdot \overline{1}$ dan $\overline{4}=\overline{4} \cdot \overline{4} \cdot \overline{4}$.

Di peroleh, $\overline{1}=\overline{4} \cdot(\overline{4} \cdot \overline{1} \cdot \overline{1}) \cdot \overline{1}=\overline{1} \cdot \overline{1} \cdot \overline{1}, \overline{1}=\overline{1} \cdot(\overline{1} \cdot \overline{1} \cdot \overline{4} \cdot) \cdot \overline{4} \cdot=\overline{1} \cdot \overline{1} \cdot \overline{1}$ yang berarti $\overline{1} \approx \overline{1}$.

Jadi, relasi transitif pada similar semu atas ring reguler stable diperumum $\mathbb{Z}_{5}$ dipenuhi.

$\overline{1} \approx \overline{6}$, berarti terdapat $\overline{1}, \overline{6} \in \mathbb{Z}_{7}$ sehingga $\overline{1}=\overline{6} \cdot \overline{6} \cdot \overline{1}, \overline{6}=\overline{1} \cdot \overline{1} \cdot \overline{6}, \overline{1}=\overline{1} \cdot \overline{1} \cdot \overline{1}$ dan $\overline{6}=\overline{6} \overline{6} \cdot \overline{6}$.

$\overline{6} \approx \overline{6}$, berarti terdapat $\overline{1}, \overline{6} \in \mathbb{Z}_{7}$ sehingga $\overline{6}=\overline{1} \cdot \overline{6} \cdot \overline{1}, \overline{6}=\overline{1} \cdot \overline{6} \cdot \overline{1}$ dan $\overline{6}=\overline{6} \overline{6} \cdot \overline{6}$.

Di peroleh, $\overline{1}=\overline{1} \cdot \overline{6} \cdot \overline{6}, \overline{6}=\overline{1} \cdot(\overline{1} \cdot \overline{1} \overline{6}) \cdot \overline{1}=\overline{1} \cdot \overline{1} \cdot 6, \overline{1}=\overline{1} \cdot \overline{1} \cdot \overline{1}$ dan $\overline{6}=\overline{6} \overline{6} \cdot \overline{6}$ yang berarti $\overline{1} \approx \overline{6}$.

Jadi, relasi serupa semu bersifat transitif pada ring reguler stable diperumum $\mathbb{Z}_{7}$.

\section{Simpulan}

Relasi serupa semu pada ring reguler stable diperumum $\mathbb{Z}_{2}, \mathbb{Z}_{2}, \mathbb{Z}_{2}$ dan $\mathbb{Z}_{2}$ bersifat refleksif, simetris dan transitif. Dengan demikian, berlaku relasi ekuivalensi pada serupa semu pada ring reguler stable diperumum.

\section{Ucapan Terima Kasih}

1. Drs. Muhammad Irfan, M.T selaku Dekan FMIPA Unsri.

2. Prof. Dr. H. Muhammad Said, M.Sc selaku Ketua Lembaga Penelitian Unsri. 


\section{Daftar Pustaka}

1. Chen H., 2000, On Generalized Stable Rings, Comm. Algebra (28), $1907-1917$.

2. Chen H, 2001, Regular Rings with Finite Stable Range, Comm. Algebra (29), 157 - 166.

3. Chen H, 2003, Generalized Stable Regular Rings, Comm. Algebra (31), 4899 - 4910.

4. Chen H, Chin A.Y.M, 2002, A Note On Regulers Rings With A Stable Range One, IJMMS.Hindawi.Com (31:7): 449 - 450

5. Goodearl K R., Menal P., 1988, Stable Range One for Rings with Many Units, J Pure Applic. Algebra (54), $261-287$

6. Goodearl K.R., 1991, Von Neumann Regular Rings, $2^{\text {nd }}$ edition, Malabar, Florida, Krieger.

7. Yuliza, E., 2010, Ring Reguler Yang Memenuhi Ring Stabil Diperumum (Generalized Stable Rings), Jurnal Penelitian Sains, ISSN: 1410-7058, Edisi Khusus Juni.

8. Yuliza E., 2013, Sifat-Sifat Similar Semu Atas Ring Reguler Stable Diperumum, Prosiding Seminar Matematika dan Pendidikan Matematika Jurusan Pendidikan Matematika UNY, ISBN: 978-979-16353-9-4.

9. Yuliza E., 2014, Ring Reguler Stable Diperumum Pada Himpunan Bilangan Bulat Modulo n, Prosiding Seminar Nasional MIPA Unsri, ISBN: 978-702-98559-1-3. 